\title{
Smart Agriculture IoT Education Course in enPiT-everi (Education Network for Practical Information Technologies - Evolving and Empowering Regional Industries) ${ }^{*}$
}

\author{
Yasunori Takemura, Keiji Kamei, Atsushi Sanada \\ Department of Life Science and System Engineering, Kyushu Institute of Technology, 2-4, Hibkinino, Wakamatsu \\ Kitakyushu, Fukuoka 808-0196, Japan \\ Department of Engineering, Nishinippon Institute of Technology, 1-11 Aratsu, Kanda, \\ Miyakogun, Fukuoka 800-0394, Japan \\ Kazuo Ishii \\ Department of Life Science and System Engineering, Kyushu Institute of Technology, 2-4, Hibkinino, Wakamatsu \\ Kitakyushu, Fukuoka 808-0196, Japan \\ E-mail: takemura@nishitech.ac.jp,kamei@nishitech.ac.jp, \\ sanada@nishitech.ac.jp, ishii@brain.kyutech.ac.jp
}

\begin{abstract}
In Japan, expectations for the utilization of IT such as AI (Artificial Intelligence) and IoT (Internet of Things) are becoming greater and greater to address the shortage of working population due to the declining birthrate and aging population, and the depopulation of rural areas. There is a serious shortage of IT human resources, especially in rural areas, and I believe that IT human resource development is an urgent task to promote the super-smart society, Society 5.0 , which is being promoted by the government. Thus, in order to promote business efficiency and productivity improvement, the utilization of IT in companies will become even more important[1].

Then, five universities, mainly in Kyushu and Chugoku regions, have been selected by the Ministry of Education, Culture, Sports, Science and Technology (MEXT) for the enPiT-Pro project, and will collaborate to provide systematic and practical IT education programs to working people from local companies, with the aim of fostering advanced IT human resources. In this paper, we report on a class of the enPIT-everi program, the Agricultural IoT Education course.
\end{abstract}

Keywords: IoT, EnPIT-everi, Agriculture, Image processing, Deep Learning, PBL education.

\section{Introduction}

In Japan, expectations for the utilization of IT such as AI (Artificial Intelligence) and IoT (Internet of Things) are becoming greater and greater to address the shortage of working population due to the declining birthrate and aging population, and the depopulation of rural areas.
There is a serious shortage of IT human resources, especially in rural areas, and we believe that IT human resource development is an urgent task to promote the super-smart society, Society 5.0, which is being promoted by the government. Thus, in order to promote business efficiency and productivity improvement, the 
utilization of IT in companies will become even more important [1].

In the midst of the global trend of the fourth industrial revolution, the Kyushu-Chugoku region has the opportunity to realize the development of new key industries that it has been seeking for many years. In order to realize this, it is necessary for the various people already working in society to adapt to the new technological innovations and grow. In order to achieve this aim, we will develop an advanced human resource development program for working people, targeting distinctive industries in the region, complementing each other in a wide range of fields through university collaboration, and incorporating the latest artificial intelligence and robotics technologies into social implementation. Then, five universities, mainly in Kyushu and Chugoku regions (the University of Kitakyushu, Kyushu Institute of Technology, Hiroshima City University, Kumamoto University and Miyazaki University), have been selected by the Ministry of Education, Culture, Sports, Science and Technology (MEXT) for the enPiT-Pro project, and will collaborate to provide systematic and practical IT education programs to working people from local companies, with the aim of fostering advanced IT human resources. In this paper, we report on one of the education courses in enPIT-everi (Education Network for Practical Information Technologies - Evolving and Empowering Regional Industries) program, the Agricultural IoT Education course.

\section{2. enPIT-everi project}

The enPIT-evri program aims to provide working adults who are already based in some industrial field with the opportunity to acquire the knowledge, skills, thinking, judgment, and organizational abilities described in the following policies by taking a systematic education program at the graduate school level, despite its short duration of 3 to 6 months. However, in consideration of the various motivations and course-taking methods of the participants, two policies for the completion of the program, "IoT Architect" and "IoT Engineer," will be established [2].

This educational program will support the development of distinctive industrial fields in the Kyushu and Chugoku regions. Companies operating in the northern part of Kyushu are interested in IoT in a wide range of industrial fields, although they are somewhat more interested in manufacturing. Therefore, this program has set up courses that take advantage of the characteristics of the five courses. The five courses are Smart Factory, Intelligent Car, Smart Life Care, Smart Agriculture, Forestry and Livestock, and Hospitality IoT.

In all courses, a case study lecture is provided as a preliminary lecture, and after that, the common lectures can be accessed via VOD (Video on Demand). Finally, there are practical laboratory exercises in each course. In this paper, we report on the "Practical laboratory exercises on agriculture" conducted as an exercise of the Smart Agriculture Course.

\section{Practical laboratory exercises on Agriculture}

In Practical laboratory exercises on agriculture, we conducted PBL-style problem-solving classes and practical assignments necessary for problem-solving. The classes are held on weekends and consist of 24 sessions (36 hours) in total. In the curriculum of the class, the following lessons were given in each lesson.

The exercises are mainly organized into four major themes: artificial intelligence exercises, image processing exercises, mechatronics (microcontroller programming) and PBL (Project Based Learning) exercises.

1. Introduction

2. Mechatronics practice 1:

3. IoT Exercise using Microcontroller 1

15. Mechatronics practice 2:

4. IoT Exercise using Microcontroller 2 Mechatronics Exercise 3:

5. IoT Exercise using Microcontroller 3

6. Artificial Intelligence Exercise 1: Introduction to Python

7. Artificial Intelligence Exercise 2:

Preprocessing of training data (Numpy)

8. Artificial Intelligence Exercise 3:

Deep Learning Recognizer

9. Artificial Intelligence Exercise 4:

Time Series Predictor

10. Artificial Intelligence Exercise 5:

Class determination using self-organizing maps [3]

11. Image Processing 1:

Development Environment for Image Processing Programs, Basics of Image Processing 
12. Image Processing 2:

Color Detection Processing (Color, Grayscale, Binary)

13. Image Processing 3:

Color Space (RGB, HSV)

14. Image Processing 4:

Counting Objects (Labeling, Center of Gravity)

15. Image processing 5:

Object detection (template matching)

16. Image processing 6:

Object detection (Hough transform)

17. Problems in agriculture, forestry and livestock industry

18. Brainstorming on agriculture, forestry and livestock industry issues

19. PBL exercise 1

20. PBL exercise 2

21. PBL exercise 3

22. PBL exercise 4

23. PBL exercise 5

24. Presentation of results and evaluation

In order to practice in the real field, we prepared an experimental field (Fig.1, 2). In the experimental field, we prepared a hydroponic field and an open-air field for IoT practice. In addition, the power supply for the exercises is secured by using solar power generation.

\subsection{IoT Data acquisition experiment using Microcontroller}

We practiced how to use a microcontroller to obtain data such as temperature, humidity, and water content in a plastic greenhouse from the cloud via the Internet. The microcontroller is an Arduino microcontroller, and the students practiced how to build a system that can obtain information from the cloud using an MQTT server.

Figure 3 shows the temperature in the greenhouse for one month, obtained by using IoT devices. The data is acquired once every 30 minutes, and can be checked on a web browser.

\subsection{Artificial Intelligent Exercise}

The students have already learned the outline of programming techniques and artificial intelligence in the common courses. Therefore, in this exercise, we used

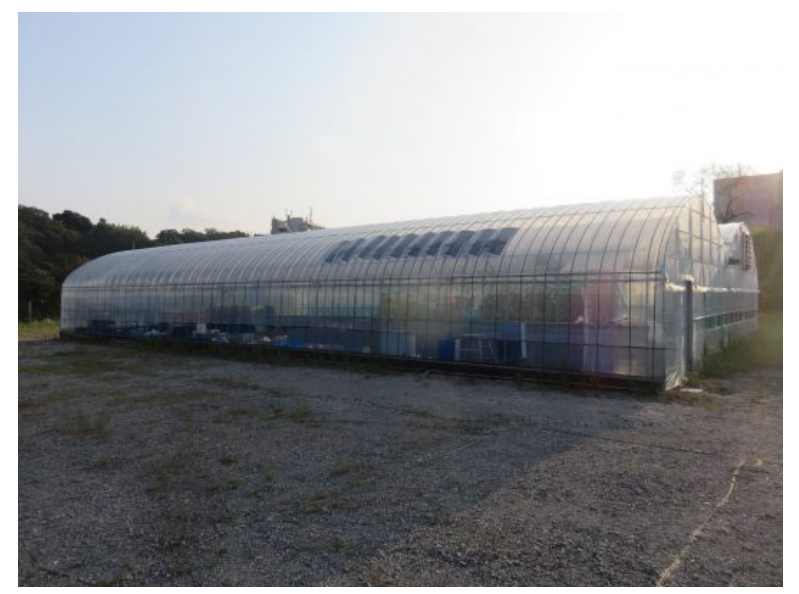

Fig.1 Experiment green house field

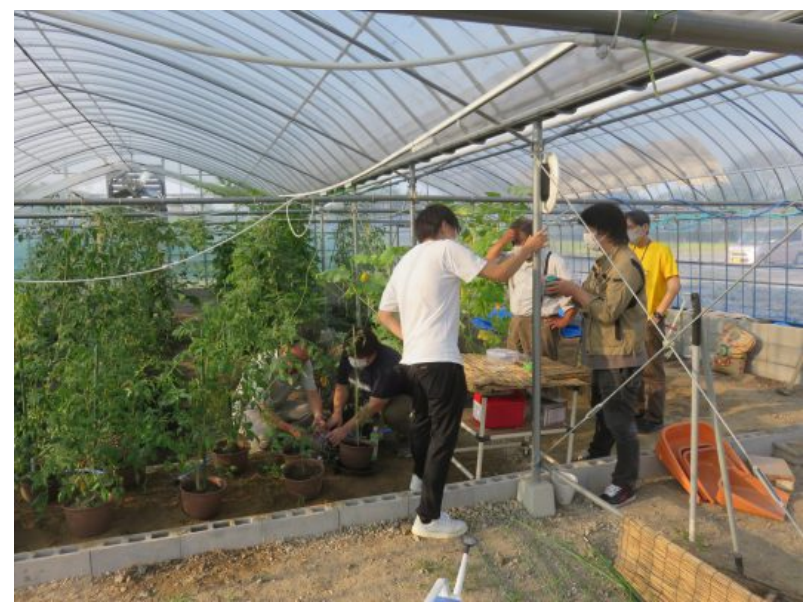

Fig.2 Scene of Experiment (Installation of IoT devices)

Fig.3 Temperature data acquired by IoT devices CNN (Convolutional Neural Network) as a method of application in a farm. CNN is capable of recognizing objects in images. Therefore, in this exercise, we created an artificial intelligence architecture to recognize caterpillars in a farm. In this exercise, we used google collaboratory.

\subsection{Image Processing exercise}

For image processing, we aimed to practice using a miniaturized computer for use in agriculture. In this exercise, we used a small computer, Raspberry $\mathrm{Pi}$, to build a system that can be equipped with $\mathrm{CNN}$ inference 


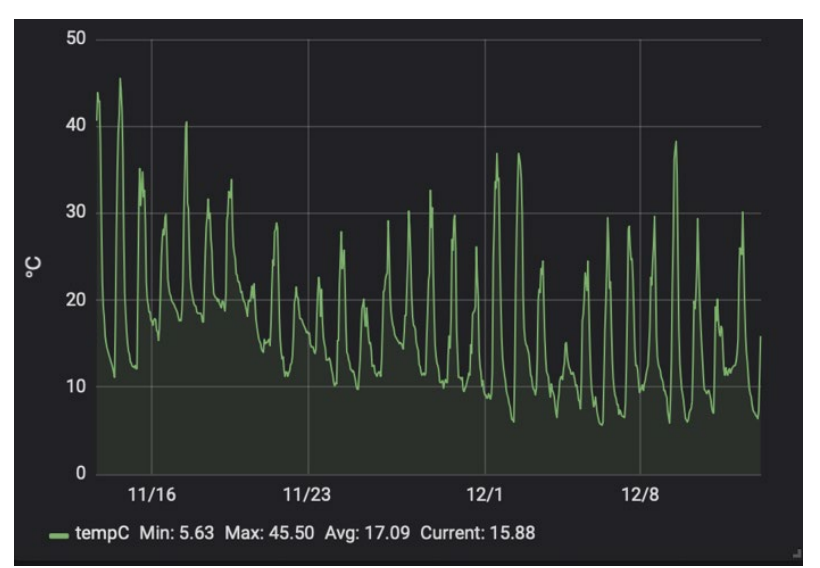

Fig.3 Temperature data in green house field

at the same time. We mainly learned about classical object recognition and filtering methods in image processing, and then performed $\mathrm{CNN}$ inference using the Neural Computing Stick.

\section{4. $P B L$ exercise}

In the PBL exercise, the students brought the equipment they had used in the past and discussed how they could use it in the company with graduate students and advisors. One of the students, who is in charge of town revitalization in the region, proposed a project that uses IoT devices to educate young people. There were many other ideas such as branding with IoT, and we were able to discuss guidelines for applying the technology in the class.

\section{Summary}

In this paper, we report on an exercise of agricultural IoT in the field of smart agriculture, which is one of the courses of enPIT-everi. In this exercise, we aimed at the development procedure of IoT devices using microcontrollers and how to apply artificial intelligence using image processing and $\mathrm{CNN}[4]$ to agriculture. In addition, we conducted a PBL exercise in which the participants, who are working people, think about how to apply the knowledge obtained in this exercise to their own work.

In the future, we would like to establish a system that enables us to carry out the exercise using an actual plastic greenhouse for a long period of time, and to obtain data for a long period of time and to use the data for inference by artificial intelligence.

\section{Acknowledgements}

This research was supported in part by enPIT-everi[1]. And, we are thankful to all participants of "Practically laboratory exercises on Agriculture" and helping students in Kyushu Institute of Technology and Nishinippon Institute of Technology

\section{References}

1. Enpit-everi homepage : "https://www.enpit-everi.jp", in Japanese

2. Enpit-everi education course introduction: "https://www.enpit-everi.jp/course/ "in japanese

3. Kohonen, Teuvo (1982). "Self-Organized Formation of Topologically Correct Feature Maps". Biological Cybernetics. Vol.43 (1): 59-69.

4. Matusugu, Masakazu; Katsuhiko Mori; Yusuke Mitari; Yuji Kaneda (2003). "Subject independent facial expression recognition with robust face detection using a convolutional neural network", Neural Networks vol.16 (5): 555-559.

(C) The 2021 International Conference on Artificial Life and Robotics (ICAROB2021), January 21 to 24, 2021 